\title{
Semi-Harmonic Scaling Enables Calculation of Masses of Elementary Particles of the Standard Model
}

\author{
Hans J. H. Geesink ${ }^{1}{ }^{*}$, Dirk K. F. Meijer ${ }^{2}$ \\ ${ }^{1}$ Ir. Previous Project Leader Nanotechnology, DSM, Geleen, The Netherlands \\ ${ }^{2}$ Pharmacokinetics and Drug Targeting, University of Groningen, Groningen, The Netherlands \\ Email:meij6076@planet.nl, *Hans.Geesink@ziggo.nl
}

How to cite this paper: Geesink, H.J.H. and Meijer, D.K.F. (2018) Semi-Harmonic Scaling Enables Calculation of Masses of Elementary Particles of the Standard Model. Journal of Modern Physics, 9, 925-947. https://doi.org/10.4236/jmp.2018.95057

Received: February 15, 2018

Accepted: April 8, 2018

Published: April 11, 2018

Copyright $\odot 2018$ by authors and Scientific Research Publishing Inc. This work is licensed under the Creative Commons Attribution International License (CC BY 4.0). http://creativecommons.org/licenses/by/4.0/

\section{Open Access}

\begin{abstract}
The underlying rules for a natural system describing cellular automata are simple, but produce highly complex behavior. A mathematical basis for the spectra of discrete coherent and non-coherent electromagnetic (EM) frequencies was derived, in which the algorithm exhibits an information distribution according to ratios of 2:3 in 1:2 at a semi-harmonic manner. This generalized music (GM) model shows that energy both in elementary particles and animate systems is semi-harmonic, quantized and discrete. A support for an ontological basis of the Standard Model was found, and indicates that the GM-model underlies the quantum field theory of subatomic particles. The present theory combines quantum mechanics and classical periodic systems, obeys to locality and solves the "hidden variable theory of Bohm". The discovered pattern of electromagnetic field eigenvalues, within a broad range of discrete frequencies, points at a de Broglie/Bohm type of causal interpretation of quantum mechanics, implying an integral resonant pilot-wave/particle modality. The model has been substantiated by a meta-analysis of measured discrete energies of: 37 different Elementary Particles, 45 different EPR-measurements, zero-point energies of elements and about 450 electromagnetic wave frequencies of cells with a mean accuracy of $0.58 \%$. It has been shown that the GM-scale is frequency-locked with zero-point oscillations, and thereby evidently implies involvement of entanglement.
\end{abstract}

\section{Keywords}

Algorithm, Elementary Particles, Coherent Wave Pattern, Electromagnetic Fields, Solitons, Harmonics, Cellular Automata, Quantum Mechanics, Standard Model, Einstein-Podolsky-Rosen, Bohm, Fröhlich, Pythagoras 


\section{Introduction}

Elementary particles are the fundamental objects of quantum field theory and are classified according to their spin and energy. The Standard Model of particle physics is the theory describing three of the four known fundamental forces (the electromagnetic, weak, and strong interactions, and not including the gravitational force) in the universe, as well as classifying elementary particles. This model is based on quantizing classical fields, like electromagnetic fields, realizing that particles basically just emerge from excitations of these fields. For example these excitations have been mathematically modelled as an infinite system of coupled quantum harmonic oscillators and the characteristic energy spectrum is given by a ladder of evenly spaced energy levels, and each level in the ladder is identified by a number $\mathrm{n}$, and the number of levels is infinite [1]. The masses of fundamental elementary particles have been calculated using the equation $\mathrm{m} / \mathrm{m}_{\text {electron }}=\mathrm{N} / 2 \alpha$, where $\alpha$ is a coupling constant of quantum electrodynamics, but $\mathrm{N}$ is an arbitrary chosen integer variable [2]. A theoretical model considers particles as electromagnetic volume resonators, capable of holding electromagnetic waves of certain frequencies, based on resonance conditions for (self-acting) nonlinear electromagnetic waves, according to de Broglie waves [3]. Although the Standard Model is believed to be theoretically self-consistent and has demonstrated successes in providing experimental predictions, it leaves some phenomena unexplained. All masses of the elementary particles are still free parameters in the Standard model, all resulting from experimental results, and a physical formula for masses of elementary particles is not yet available.

In our previous studies, a novel biophysical principle was revealed, describing an algorithm for coherent and non-coherent electromagnetic (EM) frequencies, called the GM-scale [4] [5] [6]. The particular frequency bands are scaled by an Pythagorean calculation, based on information distribution according to ratios of 2:3 in 1:2. The particular scale exhibits a core pattern of twelve eigenfrequency functions with adjacent self-similar (fractal) patterns, according to octave hierarchy. A meta-analysis on EPR-experiments learned that entanglement, achieved in continuous variable experiments is real, and can be calculated at coherent configurations of determinate EM frequencies [6].

All analysed EPR-data of the independent studies fit precisely in this derived GM-scale of coherent frequency data and turned out to be virtually congruent with the above mentioned coherent scale. A same congruence may be at stake for the distribution of masses of elementary particles by making use of the Planck-Einstein relationship:

$$
M \cdot c^{2}=h \cdot v
$$

\section{Present Postulate}

Both observations of previous EPR and biological data support the idea that the energy in quantum systems can be interpreted classically, in line with recent proposals of t'Hooft, 2016, and Dolce, 2016 on the basis of periodicity of limit 
cycles and cyclic periodicity of space-time. The latter indicates a deterministic framework of discrete frequencies that provides a causal interpretation of quantum physics. It is postulated that:

The masses of the elementary particles can be based on fixed physical parameters, due to the fact that mass is related to the Einstein-Planck relationship and a frequency scale calculated by a discrete coherent Pythagorean function: the GM-scale.

\section{A Pythagorean Function}

A mathematical basis for a spectrum of discrete coherent electromagnetic (EM) frequencies was recently derived based upon research carried out for solitons. Solitons are self-reinforcing solitary waves, that interact with complex biological phenomena such as cellular self-organization and waves in thin membranes [4] [5] [6]. The soliton model is able to describe a spectrum of electromagnetism modalities that can be applied to understand the physical principles of biological effects in living cells, as caused by endogenous and exogenous electromagnetic fields and is compatible with quantum coherence [4]. It has been found that that the first, second, and third harmonics of waves can be united within a broad range of frequencies from sub Hertz till about $10^{25} \mathrm{~Hz}$ by dividing 2:3 ratios and approximations thereof over 1:2 ratios. The spectrum of these ratios is based upon an adapted Pythagorean calculation and at this manner scales have been derived showing coherent patterns of numbers that contain a core of twelve functions that can be expressed as: $2^{0} 3^{0} 2^{\mathrm{m}}, 2^{8} 3^{-5} 2^{\mathrm{m}}, 2^{-3} 3^{2} 2^{\mathrm{m}}, 2^{5} 3^{-3} 2^{\mathrm{m}}, 2^{-6} 3^{4} 2^{\mathrm{m}}$, $2^{2} 3^{-1} 2^{\mathrm{m}}, 2^{0.5} 2^{\mathrm{m}}, 2^{-1} 3^{1} 2^{\mathrm{m}}, 2^{7} 3^{-4} 2^{\mathrm{m}}, 2^{-4} 3^{3} 2^{\mathrm{m}}, 2^{4} 3^{-2} 2^{\mathrm{m}}, 2^{-7} 3^{5} 2^{\mathrm{m}}$, in which $\mathrm{m}$ are integers [5]. The scale has been translated to Hz-frequencies for a broad range of adjacent frequency spectra for integer values of $\mathrm{m}$, of which $\mathrm{m}$ are integers, and ranges from the lowest till the highest possible frequency present in nature (Figure 1, Table 1 and Appendix 3).

A non-coherent-scale could be calculated based upon the finding that non-coherent parameters are located logarithmically just in between the coherent parameters of the 12-number scales. The derived arithmetical scales exhibit sequences of unique products of integer powers of 2, 3 and a factor $\sqrt{2}$ and contains about 1500 different determinate frequency data for ordered data and more than 1500 different numbers for disordered data in a fractal setting in both biological data, see Figure 1, and in inanimate systems. A correlation between the proposed coherent scale and the "hidden variables" as described in the theory of David Bohm has been found [6].

\section{Pilot-Wave Steering of Particles in De Broglie/Bohm Context}

Three considerations were the starting point for the search to for a deterministic quantum wave approach 1) the idea of Einstein that quantum randomness is not the determinant of the fabric of reality, 2) the conclusion of Schrödinger that 
Biological data: beneficial (green) and detrimental (red) frequencies, positioned at the GM-scale

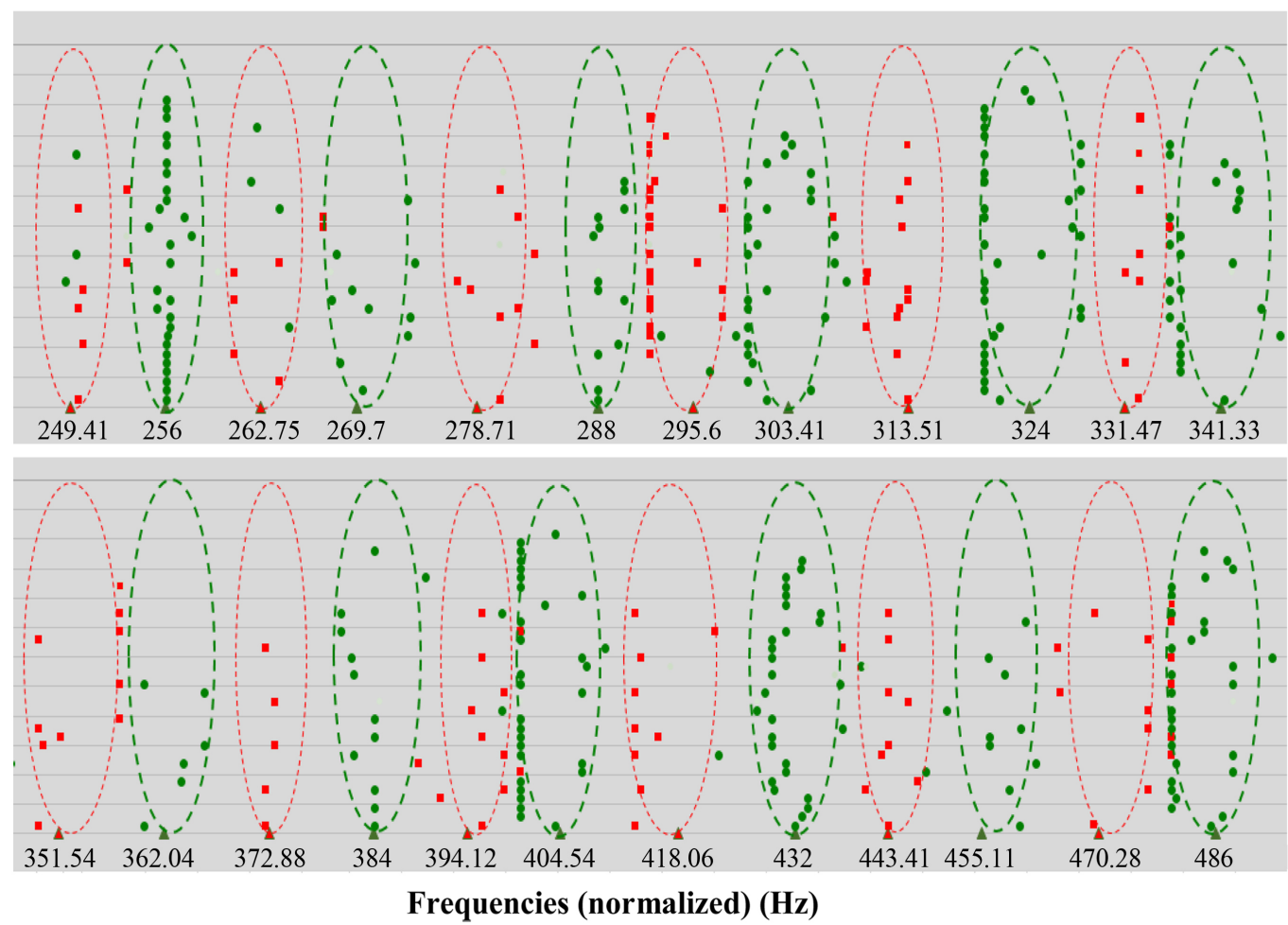

Figure 1. Measured frequency data of living cells systems that are life-sustaining (green points) and detrimental for life (in red squares) versus calculated normalized frequencies. Biological effects measured following exposures or endogenous effects of living cells in vitro and in vivo at frequencies in the bands of $\mathrm{Hz}$, $\mathrm{kHz}, \mathrm{MHz}, \mathrm{GHz}, \mathrm{THz}, \mathrm{PHz}$. Green triangles plotted on a logarithmic $\mathrm{x}$-axis represent calculated life-sustaining frequencies; red triangles represent calculated life-destabilizing frequencies. Each point indicated in the graph is taken from published biological data and are a typical frequency for a biological experiment(s). For clarity, points are distributed along the $\mathrm{Y}$-axis.

Table 1. Examples of equivalent calculated coherent 12-number frequency scales.

\begin{tabular}{rrrrrrrrrrrrrr}
\hline 256.0 & 269.70 & 288.00 & 303.41 & 324 & 341.33 & 362.04 & 384.00 & 404.54 & 432.00 & 455.12 & $486.00 \mathrm{~Hz}$ \\
1.0 & 1.0535 & 1.1250 & 1.1852 & 1.2656 & 1.3333 & 1.4142 & 1.5000 & 1.5803 & 1.6875 & 1.7778 & $1.8984 \mathrm{~Hz}$ \\
& & & & & & & & & & & & & \\
532.5 & 505.6 & 473.4 & 449.3 & 420.8 & 399.5 & 376.6 & 710.1 & 674.0 & 631.3 & 599.1 & $561.0 \mathrm{~nm}$ \\
\hline
\end{tabular}

living cells require external quantum information for their development and ecological survival, 3) the proposal of Fröhlich that living cells make use of constructive interference through so called acoustic solitons, that can be described by Bose-Einstein-statistics [7].

Louis de Broglie argued that if photons, with their wavelike properties, could be described as particles, then electrons as particles should show wave like properties with a wavelength $\lambda$, inversely proportional to their momentum $\left(p=m_{e} v\right)$ : $\lambda=h / p$ (massive particle $m_{e}$, velocity $v$, momentum $p$, Planck constant $h$ ). This relationship is now known to hold for all types of matter: all matter exhibits 
properties of both particles and waves. In his theory, particle motions are determined by a wave function, that de Broglie called a "pilot wave". For a many-body system, the pilot wave propagates in a multidimensional "configuration space", which is constructed from the co-ordinates of all the particles involved. Schrödinger developed de Broglie's idea further with a wave equation, yielding three-dimensional pulsations known as spherical harmonics [8]. The Bohmian interpretation of quantum mechanics was introduced in 1952, and later called the ontological interpretation, seen as an alternative to the standard Copenhagen interpretation. Bohm proposed an interpretation of the quantum mechanics that is nonlocal, causal, and does not treat systems and measuring apparatus differently [9] [10]. Although rejected by many, since the 1990s the interest in Bohmian mechanics has been remarkably increased [11]. The de Broglie-Bohm theory, in fact is an interpretation of non-relativistic quantum theory that postulates an actual configuration which exists even when unobserved.

According to the physicist Max Born, it is not the electrons themselves that display wave patterns, as de Broglie and Schrödinger believed, but rather the related probability distributions that indicate their likeliest positions. Although neither Schrödinger nor Einstein were comfortable with Born's statistical interpretation, it has persisted as the standard view. Einstein, Podolsky and Rosen, in 1935, originated the so-called Einstein-Podolsky-Rosen (EPR) correlation for quantum-entangled particles [12]. Two particles are entangled if the quantum states of the particles are coupled. In such a case, quantum entangled particles seem to react as a single body; there seems to be no separation in space and time.

John Bell in theory proved that the supposed non-local effect of quantum-entangled particles was probably real, and this became known as Bell's theorem or inequality. Bell's theorem ruled out local hidden variables as a viable explanation of quantum mechanics, and it left the door open for non-local hidden variables, while Schrödinger pointed out that the EPR two-particle wave function does not represent the separable form but rather of the entangled form [13] [14]. Experiments carried out to test Bell's inequality during eight decades, therefore, have led to a re-examination of the concepts of quantum mechanics, and revealed the importance of the notion of entanglement and non-locality (Reid, 2009). Many experiments have measured violation of the inferred Heisenberg uncertainty principle, and confirmed EPR-entanglement [6].

Bohm's Quantum Equilibrium has a typical determinate type of entangled configuration, of which the frequencies of the eigenvalues can be mathematically described by the GM-scale. Yet, Bohm's dynamics allow also a non-equilibrium, that are waves not in phase with the wave-functions that can be considered as non-coherent frequencies, able to disturb the proposed eigenfrequency functions. It has been shown that all physical experiments, carried out to show violation of Bell's inequality during 50 years, can be precisely located at typical coherent frequencies according to a Pythagorean scale as earlier shown for coherent states of living cells [5] and see numbers in Figure 2. 
Masses of subatomic particles can be positioned at frequency GM-scale

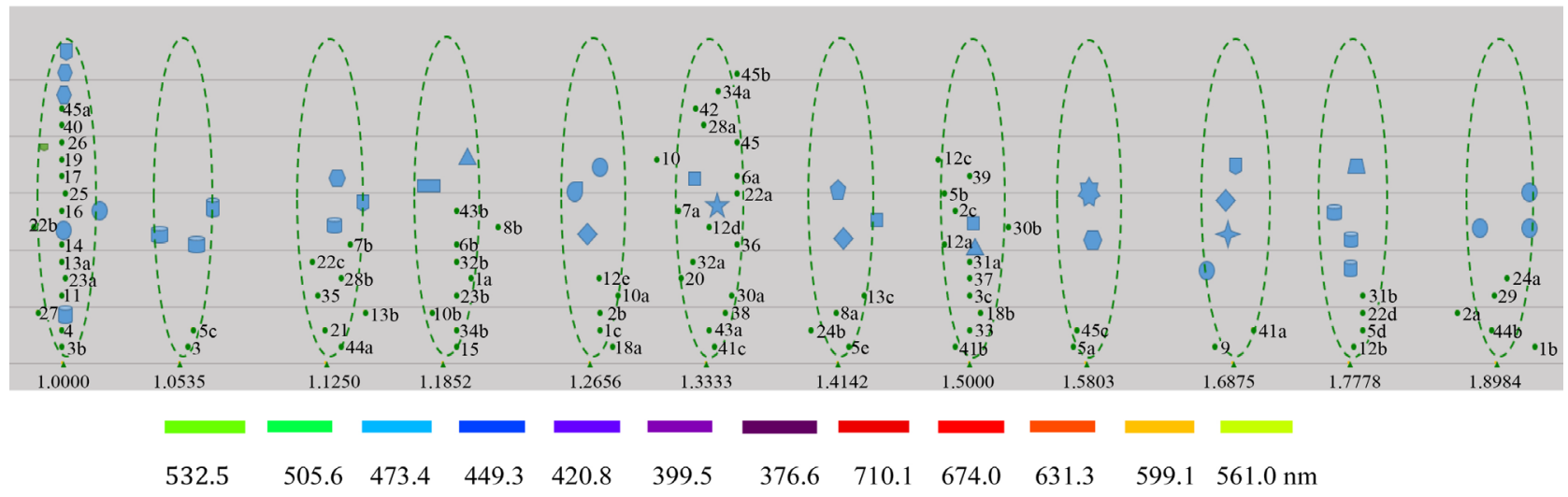

Normalized frequencies

Figure 2. Normalized frequencies of masses of Elementary Particles positioned at the GM-scale and added normalized colour spectrum. Green triangles plotted on a logarithmic x-axis represent normalized coherent-frequencies. Green points: data of Einstein-Podolsky-Rosen experiments (1972-2017). Blue points represent calculated normalized frequencies of masses, (for clarity, points are distributed along the Y-axis): Proton $\Delta$ Electron $\downarrow$ Muon $\star$ Quark $\bigcirc$ T./P. Quark $\bullet$ Baryon $\square$ Lepton $\diamond$ Neutrino 0 Boson 0 Graviton 0 Meson $\Delta$ Higgs Digamma $\Delta$ Gluon $\square$.

\section{Electromagnetic Field Frequencies in EPR Experimentation for the Study of Quantum Entanglement}

In a previous study dealing with a meta-analysis of so called EPR-experiments out of about 60 dedicated reports, we revealed that the particular EM frequencies, used to promote entangled states, also fitted the abovementioned GM-scale algorithm. The particular frequencies show a patterning very similar to those from the life systems.

An analogy with the vision of Schrödinger could be found: when you perform a Schrödinger cat experiment, and observe the superposed system, than the outcome of the cat will either be alive or be dead, but never in between. All discrete EM frequencies of the GM-scale for living organisms, show that cells are indeed either alive (sustaining coherent frequency patterns), or in contrast life deteriorating and/or life terminating (detrimental/non-coherent frequency patterns) [5] [6], but, if an effect is noticed, never in between. An extremely simple law of algorithmic coherent and non-coherent frequencies expresses this principle, as already known for Pythagorean musical scales. This law is only based on the knowledge how to divide ratios of 2:3 over ratios of 1:2 and the found rule stands for locality as well as for non-locality events (see Appendix 4 and 5).

We propose that Bohm's interpretation of quantum mechanics, that is nonlocal, causal, and based upon determinate values, is compatible with the GM-spectrum of determinate coherent frequencies. A non-equilibrium may exist when frequencies of systems are precisely located logarithmically just in between the coherent frequencies.

\section{Parameters for the Masses of Elementary Particles}

Particle physics is the branch of physics that studies the nature of the particles 
that constitute matter and radiation. The elementary particles are excitations of the quantum field that also govern their interactions. The currently dominant theory explaining these fundamental particles and fields, along with their dynamics, is called the Standard Model. It describes the strong, weak, and electromagnetic fundamental interactions, using mediating gauge bosons. Particle physics investigates the Standard Model and its various possible extensions, e.g. to the newest "known" particle, the Higgs boson, or even to the oldest known force field, gravity [15].

The dynamics of particles is described by quantum mechanics; they exhibit wave-particle duality, displaying particle-like behaviour under typical experimental conditions and wave-like behaviour in others. Following the convention of particle physicists, the term elementary particles is applied to those particles that are, according to current understanding, presumed to be indivisible and not composed of other particles [16].

The standard model introduces particle masses through a process known as spontaneous symmetry breaking caused by the Higgs field. Within this model, the mass of the Higgs gets some very large quantum corrections due to the presence of virtual particles (mostly virtual top quarks). These corrections are much larger than the actual mass of the Higgs. This means that the bare mass parameter of the Higgs in the standard model must be fine tuned in such a way that almost completely cancels the quantum corrections. According to the standard model, neutrinos are massless particles. However, neutrino oscillation experiments have shown that neutrinos do have mass. Mass terms for the neutrinos can be added to the standard model by hand, but these lead to new theoretical problems [16].

Particles of the Standard Model can be experimentally observed, and recently includes the Higgs boson [17]. Many other hypothetical elementary particles, such as the graviton, have been proposed, and have been initially observed.

John Bell in theory proved that the supposed non-local effect of quantum-entangled particles was real, and this became known as Bell's theorem or inequality. The observed correlations in Bell experiments cannot be explained in the manner Reichenbach's principle would seem to demand. Reichenbach's principle asserts that if two observed variables are found to be correlated, then there should be a causal explanation of these correlations. It has been theoretically demonstrated that under the assumption that quantum dynamics is fundamentally unitary, then it must factorize in a particular way [18]. All masses of the elementary particles are still free parameters in the Standard model, but some empirical formulae for masses of elementary particles exist. For example The Koide formula is an unexplained empirical equation discovered by Yoshio Koide [19]. It relates the masses of the three charged leptons so well that it predicted the mass of the tau:

$$
m_{e}+m \mu+m \tau=2 / 3\left(\sqrt{m_{e}}+\sqrt{m \mu}+\sqrt{m \tau}\right)^{2}
$$




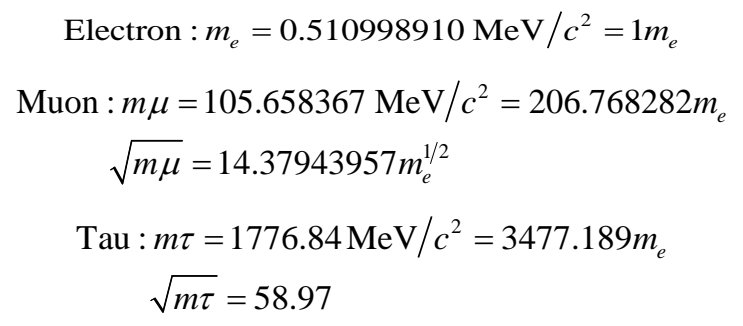

There are similar empirical formulae which relate to Quark masses that depend on the energy scale used to measure them. Taking the heaviest three quarks, charm (1290 MeV), bottom (4370 MeV) and top (174,100 MeV), gives a much closer match to get exactly $2 / 3$ within the experimental uncertainties of the masses [20]. It is of interest that the de Broglie/Conjecture underwent a significant revival in elegant hydrodynamic experimentation that revealed pilot-wave steering at the macro-scale [21]. It was shown that millimetre-sized water droplets (walkers) propel themselves along the surface of a vibrating fluid bath by virtue of resonant interaction with its own wave field. This system exhibits many features of quantum physics such as single particle diffraction, tunnelling, wave-like geometries, spin states, among others, and is very much in line with the pilot-wave theory. De Broglie pointed at the harmony of phases in which a particle vibration stays in phase with the guiding wave and maintain a state of resonance in which the particle exhibits an interaction with the underlying stochastic sub-quantum realm. A feature of walker dynamics is a sort of "path memory" due to longevity of the pilot wave. This introduces a dynamic non-local aspect of a force field induced harmonic oscillator that can also be disrupted by non-coherent field activity. The model implies a hidden-variable state that is non-local in space and time and mimics Zero Point Energy (ZPE) field character through permanent interaction with stochastic pair-production (see later Section 7).

Much of the effort to find this new physics is focused on new collider experiments. The Large Hadron Collider (LHC) was completed in 2008 to help continue the search for the Higgs boson, supersymmetric particles, and other new physics. Data on particles: gauge bosons and the recently discovered Higgs boson, leptons, quarks, mesons, neutrinos and baryon were becoming available at an increasing rate, and even measured properties of gauge bosons and the recently discovered Higgs boson, leptons, quarks, mesons, and baryons [22]. In addition, there are important non-collider experiments that also attempt to find and understand physics beyond the Standard Model.

All four fundamental forces are believed to be related, and an elegant feature is a unification of two of the four known forces into a single interaction [23]. According to Chris Neu efforts to devise a common theoretical framework that would explain the relation between the forces are perhaps the greatest goal of theoretical physicists today and the couplings of fundamental particles to the other particles are not yet all understood [23]. 


\section{The GM-Scale: Masses of Elementary Particles Are Fixed}

A causal separation of masses of elementary particles can be found, when masses are expressed in frequencies according to the Planck-Einstein relationship and positioned at the Pythagorean GM-scale. A total of about 37 independent measured masses of elementary particles ranging from sub $\mathrm{MeV}$ till $750 \mathrm{GeV}$, can be normalized to a 12 -number scale frequency scale by multiplying or dividing by multiples of 2 and positioned at the coherent-scale together with the calculated discrete coherent frequencies positioned on the x-axis (see Appendix 1 and the blue points in Figure 2). The mean deviation of the 37 data of masses, relative to the calculated different coherent reference frequencies according to the 12 -number is about $0.75 \%$, so extremely low. On the whole, a spectrum of elementary masses with a consistent pattern of frequency bands is observed.

\section{Electrodynamics and Zero-Point Field as Physical Background}

Zero-point energy (ZPE) or ground state energy is the lowest possible energy that a quantum mechanical system may have. Unlike in classical mechanics, it is estimated that quantum systems constantly fluctuate in their lowest energy state due to the Heisenberg uncertainty principle. Just as atoms and molecules, the empty space of the vacuum has these properties. According to Quantum Field Theory the universe should not be conceptualized as isolated particles, but rather as a matrix of continuous fluctuating fields. These can be seen as constituted by matter fields, whose quanta are fermions (i.e. leptons and quarks), and force fields, whose quanta are bosons (e.g. photons and gluons). All these fields have zero-point energy with a fluctuating, stochastic character since pairs of particles/antiparticles are continuously created and vanishing again. These fluctuating zero-point fields led to a kind of reintroduction in physics of a modality of "aether", and various observations such as the Casimir effect, proved the existence of this such an all pervasive energy field [24].

Stochastic electrodynamics (SED) can be seen as an extension of the de Broglie-Bohm interpretation of quantum mechanics, in which the electromagnetic zero-point field (ZPF) plays a central role as the guiding pilot-wave system for all particles as present in classical physics. The theory, therefore, represents a deterministic, nonlocal, hidden-variable theory. The "classical" dynamical model in which it is proposed that a "particle" of energy $E=h \cdot \omega$ is actually an oscillator of angular frequency $\omega$, this particle is phase-locked with the zero-point oscillations of the surrounding environment, see Figure 3. The latter field contains both regular and fluctuating components and is constrained by the boundary conditions of the experimental setup via the build-up and maintenance of standing waves [25]. SED considers the quantum properties of waves and particles as well-coordinated emergent effects, resulting from deeper (sub-quantum) nonlinear matter-field interactions [23] [24]. An underlying thesis is that all particles and fields are electromagnetic and that all particles have defined shapes 


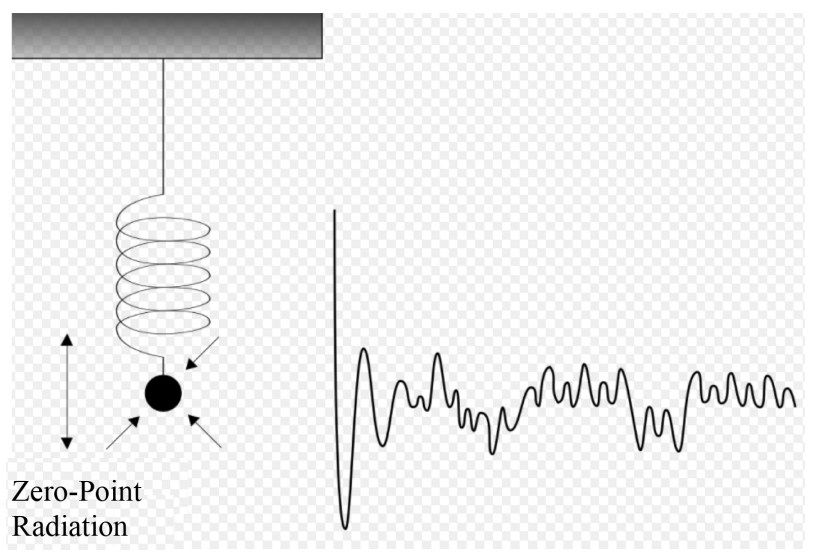

Figure 3. Zero-point radiation continually imparts random impulses on an electron, so that it never comes to a complete stop. Zero-point radiation gives the oscillator an average energy equal to the frequency of oscillation multiplied by one-half of Planck's constant (Zero-point energy of harmonic oscillator.svg).

and density profiles within three spatial dimensions as well as in the two dimensions of time and frequency [26]. The quantization of both matter and radiation field is shown to emerge as a result of the permanent interaction of matter with the ZPF. This ZPF can be represented as a homogeneous, isotropic ensemble of plane electromagnetic waves whose amplitude is equivalent to an excitation energy of $h v / 2$ of the corresponding quantized harmonic oscillator [27].

The atomic stationary states are those for which the equality holds and the energy equilibrium can hold only for certain orbital motions, due the existence of the ZPF: the electrons radiate in the field, but at the same time they absorb energy from it [28]. A classically orbiting electron in the sense of the Rutherford planetary model of 1913 would pick up energy from the ZPF at a rate exactly balancing the radiative losses due to accelerated motions of the charged electron. This balance has been demonstrated for the ground-state of the Bohr hydrogen atom. In this context the initial vibrational energy of the hydrogen molecule is equal to the vibrational zero-point energy of hydrogen, which is $0.258 \mathrm{eV}$ for $\mathrm{H} 2$ and $0.185 \mathrm{eV}$ for D2 [29].

The experimental ZPE is defined as the difference between the molecular ground state and the lowest point on its isotope-specific effective potential. The ZPE cannot be measured directly since no molecule can be observed below its ground state. Instead, the term "experimental ZPE" describes a value that is usually derived by combining experimental spectroscopic constants with standard theoretical or empirical models for anharmonic oscillators. Thus, "experimental" ZPE values are actually hybrids of experiment and theoretical [30].

If the GM-model is able to calculate both elementary particles masses and EPR-frequency states, than it should also able to predict the zero-point energies of elements, that are precisely positioned in and near the Terahertz gap, see Figure 4. Interestingly, Appendix 2 shows indeed that the zero-point energies of 


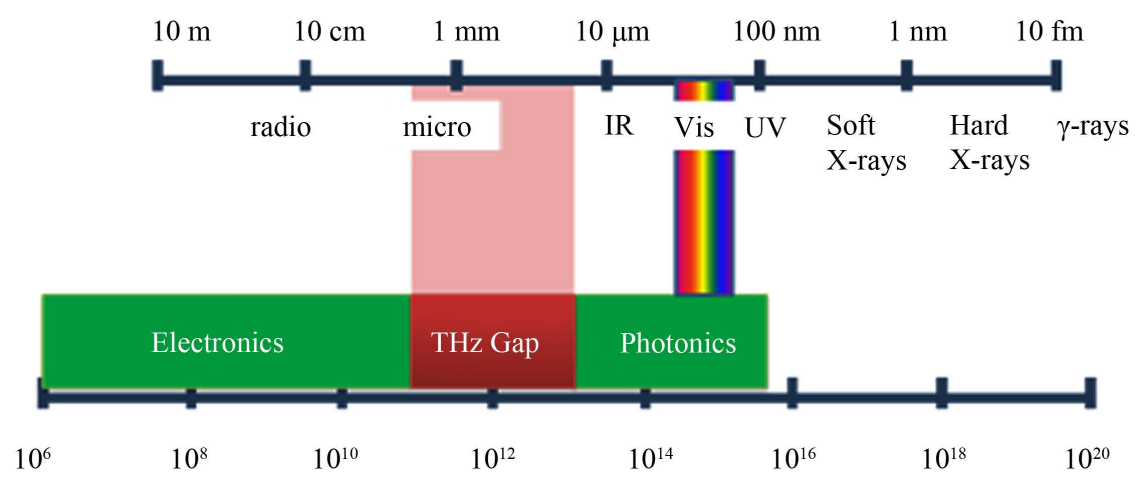

Figure 4. The terahertz gap.

elements are located at the eigenvalues of the present GM-model.

\section{Proposal for Interpretations of Quantum Mechanics and the Torus}

Elementary Cycles Theory (ECT) of Dolce (2017) postulates that every elementary "particle" of nature is characterized by persistent intrinsic space-time periodicity. In ECT the Planck energy spectrum is interpreted as an harmonic like spectrum of a mass-less periodic modalities of fundamental time periodicity $T$ (quantized energy: $E_{n}=n h \omega=n h / T$, discretized angular frequencies: $n \omega$, and time periodicity $T=h / E$ ) [31].

According to ' $t$ Hooft it is assumed that a theory describing our world starts with postulating the existence of sub-systems that, in a first approximation, evolve independently, and then are assumed to interact. It is suspected that our world can be understood by starting from a pre-quantized classical, or "ontological" system [32]. If time would be assumed to be discrete, the Hamiltonian eigenvalues would turn out to be periodic. Both theories favor a quasi-classical and quantum ontological interpretation of quantum physics, as in a primary form earlier suggested by David Bohm. Solitons are a widely observed physical phenomenon that behave like solitary waves but possess many features of particles [3]. The torus, including its knots, is seen as an attractive model for the physical description of various elementary particles [33].

Solitons are able to suppress anharmonicity (the deviation of a system from being a harmonic oscillator) by the excitation of high quantum levels, a process that facilitates the crossing of potential barriers and the transfer of a molecule to a new conformational state [4]. Particle attributes and particle conformation in space are linked, and knots can be scheduled as solitons, while toroidal solitons can be depicted as braids and framing [34]. When particles, fields, move around following classical laws, than these classical laws could resemble classical field theories such as the Navier Stokes equations and the existence of vortex and toroidal solutions [33] [35]. Also high-frequency quasi-periodic oscillations measured in a torus orbiting in the vicinity of a black hole obey to the eigenfrequencies of the proposed algorithm. According to Rezzollathe torus, in fact, can be 
thought of as a cavity in which the p modes effectively behave as trapped sound waves. If the sound speed in the cavity were constant, the frequencies of these standing waves would be in an exact integer ratio. In reality the sound speed is not constant but the eigenfrequencies found are in a sequence very close to 1:2:3:4 [36]. So cyclic energy trajectories and periodicity in quantum physics may be envisioned as recurrent spiral movements on a torus, see Figure 5.

\section{A Semi-Harmonic Quantum Oscillator and the Ontological Wave Function}

Cellular automata (CA) are discrete, abstract computational systems as general models of complexity of non-linear dynamics in a variety of scientific fields. CA-models are typically spatially and temporally discrete: they are composed of a finite or denumerable set of homogenous, simple units, the atoms or cells. John von Neumann working on self-replication has proposed a reductionist theory of biological development, trying to conceive a system capable of producing exact copies of itself [37] [38]. In 1970 the mathematician John Conway introduced Life Game [39], and in 1980s Stephen Wolfram contributed one-dimensional $\mathrm{CA}$, providing the qualitative taxonomy of their behavior [40]. Like any other CA, Life can be considered to have an initial configuration of the automatonas proposed by Turing and a suitable selection of initial conditions can ensure that the system carries out arbitrary algorithmic procedures [37]. The underlying rules for a system are simple, and even though the system is started from simple initial conditions, the behavior that the system shows can nevertheless be highly complex [39]. Finite Nature is a hypothesis that ultimately every quantity of physics, including space and time, will turn out that the amount of information in any small volume of space-time will be finite and equal to one of a small number of possibilities [37] [39]. State of the art are the use of harmonic quantum oscillators. an arbitrary potential can be approximated as a harmonic potential at the vicinity of a stable equilibrium point and one of the few quantum-mechanical systems for which an exact analytical solution is known [39]. The corresponding spectrum of energy states of a simple harmonic oscillator is:

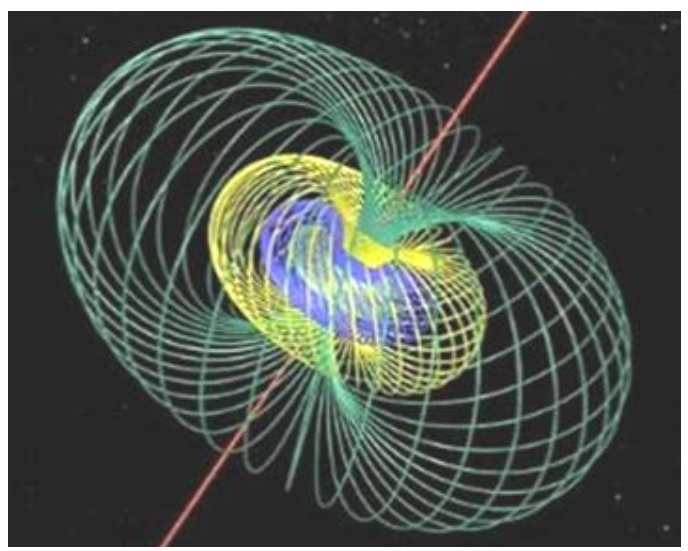

Figure 5. Toroidal geometry. 


$$
E_{n}=h \omega(n+1 / 2), \quad n=0,1,2, \cdots
$$

The energies are quantized, meaning that only discrete energy values (integer-plus-half multiples of $h \omega$ ) are possible, when a particle is confined. Secondly, the discrete energy levels are equally spaced and that the lowest achievable energy (the energy of the $n=0$ state, called the ground state) is not equal to the minimum of the potential well, but $h \omega / 2$ above it and is called zero-point energy. The current Standard Model of particle physics is based on quantizing classical fields, like electromagnetic fields, realizing that particles basically just emerge from excitations of these fields. These excitations can be mathematically modeled as an infinite system of coupled quantum harmonic oscillators and the characteristic energy spectrum is given by a ladder of evenly spaced energy levels, each level in the ladder is identified by a number $n$ and the number of levels is infinite [1] [8] [40].

In contrast the GM-model proposes a semi-harmonic coherent quantum oscillator system, in which energy levels are not equally spaced, but distributed according to a Pythagorean scale [5]. Secondly the lowest achievable energy (the energy of the so-called zero-point energy) is correlated the coherent frequencies of the ontological wave function. The proposed quantum oscillator is a basic set of three integers: $1,2,3$ and an irrational number $\sqrt{2}$, whereas integer $n\left(2^{n}\right)$ runs from the lowest possible energy to the highest possible energy. The quantum oscillator has been verified for $n=-4$ till $n=56$, by using the many mapped experiments of measured discrete energies and frequencies of: EPR-experiments, zero-point energies of elements, masses of elementary particles and frequencies of living cells [4] [5] [6]. The energy of the coherent quantum oscillator can be described by:

$$
\begin{aligned}
& E_{n}=h \omega_{\text {ref }}\left(2^{0} 3^{0} 2^{n}, 2^{8} 3^{-5} 2^{n}, 2^{-3} 3^{2} 2^{n}, 2^{5} 3^{-3} 2^{n}, 2^{-6} 3^{4} 2^{n}, 2^{2} 3^{-1} 2^{n}, 2^{0.5} 2^{n}, 2^{-1} 3^{1} 2^{n},\right. \\
& \left.2^{7} 3^{-4} 2^{n}, 2^{-4} 3^{3} 2^{n}, 2^{4} 3^{-2} 2^{n}, 2^{-7} 3^{5} 2^{n}\right) \\
& \omega_{r e f}=1 \text { and } n=<-4 \text { till }>52 .
\end{aligned}
$$

The ontological wave function describes the entangled combinations of coherent templates, represented by 12 eigenvalue functions, see Equation (2). The template states and combinations thereof form a very subset of the ontological state and have non-trivial space-like correlations, while the ontological operators show symmetry to the quantum operators for al n's. The vacuum state will contain the most elementary ontological state, while the real world is assumed to be in a superposition of the template states. It is considered that the waves of the quantum operators are located at toroidal surfaces.

\section{Conclusions}

The GM-model shows that Einstein was right, when he objected against the conclusions drawn by Bohr and Heisenberg: there is no randomness in nature. Elements of statistical probability distributions from the basic laws of quantum mechanics are unnecessary. The GM-model calculates discrete EM frequency values that are entangled and positioned at coherent, non-coherent (or chaotic) 
scales and mathematically derived according to an actualized Pythagorean scaling [5].

The GM-model is able to calculate:

1) The location of masses of elementary particles (Proton, Electron, Quarks, Leptons, Bosons, Higgs, Digamma, Graviton) having energies from sub $\mathrm{MeV}$ till $750 \mathrm{GeV}$, with a mean accuracy of $0.75 \%$.

2) Locations of zero-point energies of elements.

3) Eigen frequencies of Einstein Podolsky Rosen-systems, with a mean accuracy of $0.65 \%$.

4) In principle toroidal eigenfrequencies of blackholes.

5) Eigen frequency patterns of living cells, with a mean accuracy of $0.38 \%$.

It can be concluded therefore that the GM-scale and the experimental realizations ad 1), 2), 3) and 4) provide a way to demonstrate a type of entanglement inextricably connected with quantum non-locality, phase and frequency-locked with zero-point oscillations, and always imply entanglement [5] [6].

The theoretical background of the found measured regularities of patterns in inanimate and animate materials/particles has been based upon a mathematical scale that shows a high or even the highest possible coupling of numbers at a coherent way. This indicates that nature organizes its components at a highly coherent semi-harmonic way. The latter is adequately expressed by a semi-harmonic scale operating within a broad range of coherence inducing frequencies from sub Hertz till larger than YHz. Therefore, the 12-number scale describing these features is tentatively called a universal scale. A scale of decoherent frequencies has been derived, located logarithmically just in between the calculated coherent frequencies, which stands for decay in living cells, and decay of inanimate matter probably into a Van der Waals gas, which is quantum mechanical stochastic.

In conclusion, quantum mechanics of the investigated inanimate and animate systems are based on a deterministic principle, in line with the recent theories of 't Hooft [32] [33], Sbitnev [34], and Dolce [35].

According to the present work, such a deterministic modality can be described by an underlying mechanism based upon a semi-harmonic quantum oscillator that is evidently instrumental in the unification of the first, second, and third harmonics, as described by a Pythagorean descending scale and octave hierarchy. This deterministic system operates in a dynamic context due to the ZPE information space that intervenes at the same coherent frequencies. An interpretation of a non-relativistic quantum mechanics has been proposed, related to a natural quantum oscillator, based on analysis of energies of 37 different elementary particles, EPR-measurements of about 40 different scientific groups in the period 1972-2017, analysis of zero-point energies of elements, toroidal eigenfrequencies of blackholes, and 500 biological studies related to electromagnetic frequencies of cells in the period 1950 till now.

The present study confirms the generalized character of the biophysical prin- 
ciple, coined by us the GM-scale. It may also provide a non-empirical foundation for the standard model of wave/particles and through its wide spectrum of eigenfrequencies, the potential for down scaling of yet undiscovered wave/particle modalities down to string and Planck scales.

\section{References}

[1] Rashid, M.A. (2006) Transition Amplitude for Time-Dependent Linear Harmonic Oscillator with Linear Time-Dependent Terms Added to the Hamiltonian. Proceedings of the 12th Regional Conference on Mathematical Physics, Islamabad, Pakistan, 27 March-1 April 2006, 39-46. https://doi.org/10.1142/9789812770523_0006

[2] Greulich, K.O. (2010) Calculation of the Masses of All Fundamental Elementary Particles with an Accuracy of Approx. 1\%. Journal of Modern Physics, 1, 300-302. http://www.SciRP.org/journal/jmp https://doi.org/10.4236/jmp.2010.15042

[3] Kyriakos, A.G. (2011) Nonlinear Theory of Elementary Particles Part XV: On Calculation of Elementary Particles' Masses. Prespacetime Journal, 2, 1933-1948.

[4] Geesink, J.H. and Meijer, D.K.F. (2017) Bio-Soliton Model that Predicts Non-Thermal Electromagnetic Frequency Bands, that Either Stabilize Living Cells. Electromagnetic Biology and Medicine, 36, 357-378. https://doi.org/10.1080/15368378.2017.1389752

[5] Geesink, J.H. and Meijer, D.K.F. (2018) Mathematical Structure for EMF Life Algorithm, That May Reflect Bohm's Implicate Order. Journal of Modern Physics, 9, 851-897.

[6] Geesink, J.H. and Meijer, D.K.F. (2018) A Semi-Harmonic Electromagnetic Frequency Pattern Organizes Non-Local States and Quantum Entanglement in both EPR Studies and Life Systems. Journal of Modern Physics, 9, 898-924.

[7] Fröhlich, H. (1969) Quantum Mechanical Concepts in Biology. In: Marois, M., Ed., Theoretical Physics to Biology, North-Holland, Amsterdam, The Netherlands, 13-22.

[8] Schrödinger, E. (1935) Mathematical Proceedings of the Cambridge Philosophical Society, 31, 555-563. https://doi.org/10.1017/S0305004100013554

[9] Bohm, D. (1952) A Suggested Interpretation of the Quantum Theory in Terms of "Hidden" Variables, I \& II. Physical Review, 85, 166-179. https://doi.org/10.1103/PhysRev.85.166

[10] Bohm, D. and Hiley, B.J. (1975) On the Intuitive Understanding of Nonlocality as Implied by Quantum Theory. Foundations of Physics, 5, 93-109.

https://doi.org/10.1007/BF01100319

[11] Sanz, S. (2017) Bohm's Approach to Quantum Mechanics: Alternative Theory or Practical Picture? arXiv:1707.00609v1 [quant-ph]

[12] Einstein, A., Podolsky, B. and Rosen, N. (1935) Physical Review, 47, 777. https://doi.org/10.1103/PhysRev.47.777

[13] Bell, J.S. (1986) Beables for Quantum Field Theory. Physics Reports, 137, 49-54. https://doi.org/10.1016/0370-1573(86)90070-0

[14] Bell, J.S. (1988) Speakable and Unspeakable in Quantum Mechanics. Cambridge University Press, Cambridge.

[15] Braibant, S., Giacomelli, G. and Spurio, M. (2009) Particles and Fundamental In- 
teractions: An Introduction to Particle Physics. Springer, Berlin, 313-314.

[16] Lykken, J.D. (2010) Beyond the Standard Model. CERN Yellow Report. CERN, 101-109. arXiv:1005.1676. (Physics beyond the Standard Model, Wikipedia)

[17] Khachatryan, V., et al. (2012) Observation of a New Boson at a Mass of $125 \mathrm{GeV}$ with the CMS Experiment at the LHC. Physics Letters B, 716, 30-61. https://doi.org/10.1016/j.physletb.2012.08.021

[18] Allen, J.M.A., Barrett, J., Horsman, D.C., Lee, C.M. and Spekkens, R.W. (2017) Quantum Common Causes and Quantum Causal Models. Physical Review X, 7, Article ID: 031021. https://doi.org/10.1103/PhysRevX.7.031021

[19] Koide, Y. (1982) Fermion-Boson Two Body Model of Quarks and Leptons and Cabibbo Mixing. Lettere al NuovoCimento, 34, 201-205. https://doi.org/10.1007/BF02817096

[20] Cao, F.G. (2012) Neutrino Masses from Lepton and Quark Mass Relations and Neutrino Oscillations. Physical Review D, 85, Artcile ID: 113003.

[21] Bush, J.W.M. (2015) The New Wave of Pilot-Wave Theory. Physics Today, 68, 47. https://doi.org/10.1063/PT.3.2882

[22] Patrignani, C., et al. (2017) Review of Particle Physics, 2016-2017. Chinese Physics $C, 40$, Article ID: 100001.

[23] Neu, C. (2013) Lecture: The Interplay between the Top Quark and the Higgs Boson. Department of Physics, University of Virginia, Charlottesville. http://www.phys.virginia.edu/Announcements/Seminars/Slides/S2628.pdf

[24] Davies, P.C.W. (2011) Out of the Ether: The Changing Face of the Vacuum. New Scientist, 212, 50-52. https://doi.org/10.1016/S0262-4079(11)62858-3

[25] Gross, G. (2014) Ab Initio Quantum and Molecular Dynamics of the Dissociative Adsorption of Hydrogen on Pd(100). International Journal of Modern Physics B, 28, Article ID: 1450179. https://doi.org/10.1142/S0217979214501793

[26] Underhill, M.J. (2013) Electromagnetic Structures and Inertias of Particles including the Higgs Boson. Progress in Electromagnetics Research Symposium Proceedings, Taipei, 25 March 2013.

[27] Ibison, M. and Haisch, B. (1996) Quantum and Classical Statistics of the Electromagnetic Zero-Point Field. Physical Review A, 54, 2737-2744.

[28] De la Peña, L., Cetto, A.M. and Valdés-Hernandes, A. (2014) The Zero-Point Field and the Emergence of the Quantum. International Journal of Modern Physics E, 23, Article ID: 1450049. https://doi.org/10.1142/S0218301314500499

[29] Gross, A. (1998) Reactions at Surfaces Studied by Ab Initio Dynamics Calculations. Surface Science Reports, 32, 291-340.

[30] Irikura, K.K. (2007) Experimental Vibrational Zero-Point Energies: Diatomic Molecules. Journal of Physical and Chemical Reference Data, 36, 389. https://doi.org/10.1063/1.2436891

[31] Dolce, D. (2017) Introduction to the Quantum Theory of Elementary Cycles: The Emergence of Space, Time and Quantum. arXiv:1707.00677v1 [physics.gen-ph]

[32] ' $t$ Hooft, G. (2007) A Mathematical Theory for Deterministic Quantum Mechanics Journal of Physics. Conference Series, 67, Article ID: 012015. https://doi.org/10.1088/1742-6596/67/1/012015

[33] 't Hooft, G. (2016) Fundamental Theories of Physics, The Cellular Automaton Interpretation of Quantum Mechanics. Vol. 185, Springer International Publishing, New York. 
[34] Avrin, J.S. (2012) Knots on a Torus: A Model of the Elementary Particles. Symmetry, 4, 39-115. https://doi.org/10.3390/sym4010039

[35] Sbitnev, V.I. (2017) Hydrodynamics of Superfluid Quantum Space: de Broglie Interpretation of the Quantum Mechanics. Quantum Studies. Mathematics and Foundations. https://doi.org/10.1007/s40509-017-0116-Z

[36] Rezzolla, L., Yoshida, S., Maccarone, T.J. and Zanotti, O. (2003) A New Simple Model for High-Frequency Quasi-Periodic Oscillations in Black Hole Candidates. Monthly Notices of the Royal Astronomical Society, 344, L37-L41. https://doi.org/10.1046/j.1365-8711.2003.07018.x

[37] Francesco, B. and Jacopo, T. (2017) Cellular Automata. In: Zalta, E.N., Ed., The Stanford Encyclopedia of Philosophy.

https://plato.stanford.edu/archives/fall2017/entries/cellular-automata/

[38] Von Neumann, J. (1951) The General and Logical Theory of Automata. In: Jeffress, L.A., Ed., Cerebral Mechanisms in Behavior. The Hixon Symposium, John Wiley \& Sons, New York, 1-41.

[39] Buchmüller, W. and Lüdeling, C. (2006) Field Theory and Standard Model. arXiv:hep-ph/0609174.

[40] Wolfram, S. (1983) Statistical Mechanics of Cellular Automata. Reviews of Modern Physics, 55, 601-644. https://doi.org/10.1103/RevModPhys.55.601 


\section{Appendix 1. References about Measured Masses of Elementary Particles}

1) Olive, K.A. et al. (2014) "Review of Particle Physics". Chinese Physics C. 38 (9): 090001. Bibcode: 2014ChPhC.38i0001O. doi:10.1088/1674-1137/38/9/090001.

2) Patrignani, C. et al. (2017) Particle Data Group, Chin. Phys. C, 40, 100001 (2016) and 2017 update.

3) Grönqvist, H. (2012) Particle physics models with four generations.

4) Fuster, J., Irles, A., Vos, M., Uwer, P. (2012) The Higgs Boson, ISBN: 978-1-466824133, $2012>180 \mathrm{EV}$ top quark, Humboldt Universität, Berlin S. Alioli Ernest Orlando Lawrence BNL U. of California.

5) LHCb collaboration (2014). "Observation of the resonant character of the Z(4430)-state”. Physical Review Letters. 112 (22). arXiv:1404.1903v1.

6) Lü, Q.F., Wang, X.Y., Xie, J. J., Chen, X.R., Dong, Y.B. (2016). Neutral hidden charm pentaquark states $\mathrm{P} 0 \mathrm{c}(4380)$ and $\mathrm{P} 0 \mathrm{c}(4450)$ in the $\pi-\mathrm{p} \rightarrow \mathrm{J} / \psi \mathrm{n}$ reaction. Phys. Rev. D 93, 034009.

7) Wang, Z.G., (2016) Analysis of the $3 / 2$ pentaquark states in the diquark-diquark-antiquark model with QCD sum rules, Nuclear hysics B Volume 913, Pages 163-208, https://doi.org/10.1016/j.nuclphysb.2016.09.009.

8) Karliner, M., Rosner, J.L. (2017) Quark-level analogue of nuclear fusion with doubly heavy baryons, Nature 551, 89-91, DOI: 10.1038/nature24289.

9) Frandsen, M.T., Masina, I., Sanninor, F. (2009) Fourth Lepton Family is Natural in Technicolor. arXiv:0905.1331v1 [hep-ph].

10) Nakamura, K. et al. (2010) Particle Data Group, J.Phys. G 37, 075021.

11) Beringer, J. et al. (2012) Particle Data Group, PR D86, 010001 (2012) (URL: http://pdg.lbl.gov).

12) Kornowski, S. (2017) The Origin of the $Z$ and $W$ Bosons.

13) Von Buddenbrock S. (2017) PRODUCTION OF THE MADALA BOSON IN ASSOCIATION WITH TOP QUARKS Wits HEP group SAIP.

14) Abbott, B.P. et al. (2017) LIGO Scientific Collaboration and Virgo Collaboration. "GW170104: Observation of a 50-Solar-Mass Binary Black Hole Coalescence at Redshift 0.2". Physical Review Letters. 118: 221101, Bibcode: 2017PhRvL.118v1101A.

15) Bogdanowicz, J., Ciok, P., Galster, Z., Saniewska, T., Zielińsk, P. (1963) Nuclear Physics, Volume 40, January, Pages 270-281.

16) Murray, W. (2016) The sensitivity of the Higgs boson branching ratios to the $\mathrm{W}$ boson width, arXiv:1604.01665v1 [hep-ph].

17) Gherghetta, T., Von Harling, B., Medina, A.D., Schmidt, M.A. (2013) The Scale-Invariant NMSSM and the $126 \mathrm{GeV}$ Higgs Boson, arXiv: 1212.5243v2 [hep-ph].

18) Petersson, C., Torre, R. (20160 $750 \mathrm{GeV}$ Diphoton Excess from the Goldstino Superpartner, Phys. Rev. Lett. 116, 151804.

19) Burgio, G., Quandt, M., Reinhardt, H. (2009) Coulomb-gauge gluon propagator and the Gribov formula. Phys Rev Lett. Jan 23; 102(3):032002.

20) Carrasco N. et al. (2014) Up, down, strange and charm quark masses with 
$\mathrm{Nf}=2+1+1$ twisted mass lattice QCD. arXiv:1403.4504v3 [hep-lat].

21) Harland-Lang, L.A., Martin, A.D., Motylinski, P., Thorne, R.S. (2016). Charm and beauty quark masses in the MMHT2014 global PDF analysis. Eur. Phys. J., 76:10. DOI 10.1140/epjc/s10052-015-3843-5.

\section{Masses of Elementary Particles}

Mass and energy are essentially equivalent $(E=m c 2)$, and so the unit of energy is also a unit of mass. The energy of a wave with a given wavelength or frequency can be expressed by Planck's law $E=h v$. The value of this constant is $h=$ $4.135669 \times 10^{-21} \mathrm{MeV}$ sec. Both equations set the scale for quantum phenomena, and therefor mass can be expressed in a frequency: $m=h v / c 2$.

\section{Quarks}

$\mathrm{m}_{\mathrm{u}}=2.3 \mathrm{MeV}>2,300,000 \mathrm{eV}>556,137,550.008 \mathrm{THz}>2.0718 \mathrm{THz}>$ alg. ref. $2.0873 \mathrm{Thz}>$ delta $0.74 \%>$ ref. $1.8984 \mathrm{~Hz}>1.8844 \mathrm{~Hz}$

$\mathrm{m}_{\mathrm{d}}=4.7 \mathrm{MeV}$ (Patrignani) $>1,136,454,993.496 \mathrm{THz}>1.05840 \mathrm{THz}>$ alg. Ref. $1.0437 \mathrm{Th}>$ delta $1.41 \%>$ alg. Ref. $1.8984 \mathrm{~Hz}>1.9252 \mathrm{~Hz}$

Charm Quark (Harland-Lang, 2016) $1.2 \mathrm{GeV}>290,158,721,743.820 \mathrm{THz}>$ $2.1112 \mathrm{THz}>$ alg. $2.0873 \mathrm{Thz}>$ delta $1.15 \%>\operatorname{ref} 1.8984 \mathrm{~Hz}>1.9202 \mathrm{~Hz}$

$\mathrm{m}_{\mathrm{b}}=4.18 \mathrm{GeV}$ (Patrignani) > 1,010,719,547,407.64 THz > $1.8385>$ alg.

1.8554 , delta $0.91 \%>$ alg. $1.6875 \mathrm{~Hz}>1.6721 \mathrm{~Hz}$

$\mathrm{m}_{\mathrm{t}}=160 \mathrm{GeV}$ (Patrignani) $>38,687,829,565,842.68 \mathrm{THz}>1.0996 \mathrm{Thz}>$ alg.

$1.0995 \mathrm{Thz}>$ delta $0.01 \%>1.0001 \mathrm{~Hz}$

$\mathrm{m}_{\mathrm{c}}=1.27 \mathrm{GeV}$ (Patrignani): $>307,084,647,178.87 \mathrm{THz}>1.1172 \mathrm{THz}>$ alg. $1.0995 \mathrm{Thz}>$ delta $1.61 \%>$ alg $1.0 \mathrm{~Hz}>1.0161 \mathrm{~Hz}$

$\mathrm{m}_{\mathrm{s}}=99.6 \mathrm{MeV}$ (Carrasco): $>24,083,173,904.737 \mathrm{THz}>1.4018 \mathrm{THz}>$ ref $1.3915 \mathrm{Thz}$. Delta $0.74 \%>\operatorname{ref} 1.2656 \mathrm{~Hz}>1.2750 \mathrm{~Hz}$

\section{Top quarks}

$80 \mathrm{GeV}$ (Fuster et al. 2012) > 43,523,808,261,573.02344 THz > 1.23702 THz > alg. 1.23702 , delta $0 \%>1.1250 \mathrm{~Hz}$

$170 \mathrm{GeV}>$ (Fuster et al. 2012) 41,105,818,913,707.851 Thz $>1.1683 \mathrm{THz}>$ alg. $1.1583 \mathrm{Thz}>$ delta $0.86 \%>1.0626 \mathrm{~Hz}$

$160 \mathrm{GeV}>($ Fuster et al. 2012) > 38,687,829,565,842.68 THz > $1.0996 \mathrm{THz}>$ alg. $1.0995>$ delta $0.01 \%>1.0001 \mathrm{~Hz}$

$\mathrm{m}_{\mathrm{t}}=171 \pm 2 \mathrm{GeV}>41,347,617,848,494.36 \mathrm{THz}>1.1751 \mathrm{THz}>$ alg $1.1583>$ delta $1.45 \%$ (Kühn) > alg. $1.0535>1.0688 \mathrm{~Hz}$

$\mathrm{m}_{\mathrm{c}}=1.3 \mathrm{GeV}$ (Grönqvist, 2012) $>314,338,615,222.5 \mathrm{THz}>1.1436 \mathrm{THz}>$ ref. $1.1583 \mathrm{Th}>$ delta $1.27 \%>$ ref $\mathrm{Hz} 1.0535>1.0401 \mathrm{~Hz}$

\section{Tetraquark}

Z $4430 \mathrm{MeV}$ (LHCb col., 2014) > 1,071,169,281,104.26 THz > 1.9484 THz > ref. $1.9547 \mathrm{THz}>$ delta $0.32 \%>$ ref. $1.7778 \mathrm{~Hz}>1.7721 \mathrm{~Hz}$

\section{Pentaquark}

$4.45 \mathrm{GeV}(\mathrm{Lü}, 2016)>1,076,005,259,799.99 \mathrm{THz}>1.9572 \mathrm{THz}>$ alg. 1.9547 $\mathrm{THz}$, delta $0.13 \%>$ ref. $\mathrm{Hz} 1.7778 \mathrm{~Hz}>1.7801 \mathrm{~Hz}$

4.39 GeV (Zhi-Gang Wang, 2016) > 1,061,497,323,712.808 Thz > alg. 1.9308 Thz > ref. alg. $1.9547>$ delta $1.22 \%>1.7561 \mathrm{~Hz}$ 


\section{Baryons}

$1650 \mathrm{MeV}$ (Patrignani et al, 2016) > 398,968,242,397.75 THz > $1.4514 \mathrm{THz}>$ ref. $1.4660 \mathrm{THz}>$ delta $0.99 \%>$ ref. $1.3333 \mathrm{~Hz}>1.3201 \mathrm{~Hz}$

$3621 \mathrm{MeV}$ (Karliner \& Rosner 2017) 875,553,942,861.97 Thz > 1.5926 THz > ref. $1.5549>$ delta $2.43 \%>$ ref. $1.4142 \mathrm{~Hz}>1.4486 \mathrm{~Hz}$

N Baryon Mp: 938.272081 MeV (Patrignani et al., 2016) > 226,873,189,725.728 $\mathrm{THz}>1.6506 \mathrm{Thz}>$ ref. $1.6493>$ delta $0.08 \%>\operatorname{ref} 1.5 \mathrm{~Hz}>1.5012 \mathrm{~Hz}$

\section{Lepton}

100.8 GeV (Frandsen, 2009) > 24,373,332,626,480.89 THz $>1.3854 \mathrm{THz}>$ alg. $1.3915>$ delta $0.44 \%>$ alg $1.2656 \mathrm{~Hz}>1.260 \mathrm{~Hz}$

\section{Neutrino}

45.0 GeV(Nakamura, K. et al., 2010) > 10,880,952,065,393.25 THz > 1.237 $\mathrm{THz}>1.2370 \mathrm{THz}>$ delta $0 \%>1.1250 \mathrm{~Hz}$

$39.5 \mathrm{GeV}$ (Nakamura, K. et al., 2010) for Majorananeutrino > 9,551,057,924,067.414 THz > 1.0858 THz > alg.1.0995 Thz > delta 1.25\%> $0.9875 \mathrm{~Hz}$

$80.5 \mathrm{GeV}$ (Nakamura, K. et al., 2010, Frandsen, 2009) for Majorananeutrino > $19,464,814,250,314.60 \mathrm{Thz}>1.1064 \mathrm{THz}>$ alg. $1.0995 \mathrm{THz}>$ delta $0.63 \%>$ $1.0063 \mathrm{~Hz}$

$\mathrm{m}_{\mathrm{t}}$ Lepton (Patrignani et al.) 1776.86 MeV > 429,642,855,264.77 THz $>1.5630$ $\mathrm{THz}>$ ref. $1.5549>$ delta $0.52 \%>$ ref. $1.4142 \mathrm{~Hz}>1.4216 \mathrm{~Hz}$

Tau neutrino $15.5 \mathrm{MeV}>3,747,883,489.191 \mathrm{THz}>1.7452 \mathrm{Thz}>$ ref. 1.7376

$\mathrm{THz}>$ delta $0.44 \%>$ ref. $1.5803 \mathrm{~Hz}>1.5873 \mathrm{~Hz}$

$\mathrm{m}_{\mathrm{e}}$ Lepton (Patrignani et al.) $0.5109989461 \mathrm{MeV}>123,558,989.697 \mathrm{THz}>$ $1.8412 \mathrm{THz}>$ ref. $1.8554>$ delta $0.77 \%>$ ref. $1.6875 \mathrm{~Hz}>1.6745 \mathrm{~Hz}$

\section{Boson}

Z Boson 91.1875 GeV (Beringer, 2012) > 22,049,161,265,312.89 THz > 1.2533

$\mathrm{THz}>$ alg. $1.2370 \mathrm{THz}$ delta $1.32 \%$ ref alg $1.1250 \mathrm{~Hz}>1.1399 \mathrm{~Hz}$

W Boson 80.385 GeV (Kornowski, 2017) > 19,437,007,372,814.15 THz > $1.10486 \mathrm{THz}>$ alg $1.0995>$ delta $0.49 \%>\operatorname{ref} 1.0000 \mathrm{~Hz}>1.0049 \mathrm{~Hz}$

Madala Boson 270 GEV (Buddenbrock, 2017) > 65,285,712,392,359.53 THz > $1.8555 \mathrm{THz}>$ alg. 1.8554 delta $0 \%>1.6875 \mathrm{~Hz}$

\section{Graviton}

$7.7 \times 10^{-23} \mathrm{eV} / \mathrm{c}^{2}($ Abbott, 2017) $>1.4544 \mathrm{eV}>351.67 \mathrm{THz}>1.3737 \mathrm{THz}>$ alg 1.3915 , delta $1.27 \%$, alg ref $1.2656 \mathrm{~Hz}>1.2495 \mathrm{~Hz}$

\section{Meson}

139.57018 MeV (Patrignani) > 33,747,920,851.96 THz > 1.9644 THz > ref. $1.9547>$ delta $0.50 \%>$ ref. $1.7778 \mathrm{~Hz}>1.7867 \mathrm{~Hz}$

\section{Proton}

$0.938 \mathrm{GeV}>226,807,400,829.75 \mathrm{THz}>1.6502 \mathrm{THz}>$ alg. $1.6493 \mathrm{THz}>$ delta $0.06 \%>$ alg. $1.5000 \mathrm{~Hz}>1.4991 \mathrm{~Hz}$

\section{Proton interaction}

$24 \mathrm{GeV}($ Bogdanowicz 1963) > 5,803,174,434,876.40 THz $>1.3195 \mathrm{THz}>$ ref $1.3031 \mathrm{Thz}$, delta $1.26 \%>\operatorname{ref} 1.1852 \mathrm{~Hz}>1.2001 \mathrm{~Hz}$ 


\section{Electron}

$0.000511 \mathrm{Gev}>123,559,255.67 \mathrm{THz}>1.8412 \mathrm{THz}>$ alg. $1.8554 \mathrm{THz}$ delta $0.77 \%>$ alg ref $1.6875 \mathrm{~Hz}>1.6745 \mathrm{~Hz}$

Higgs particle

$126 \mathrm{GeV}$ (Gherghetta, 2013, Murray 2016) > 30,466,665,783,101.1 THz > $1.7318 \mathrm{THz}>$ alg 1.7376 delta $0.3 \%$ > alg. Ref. $1.5803 \mathrm{~Hz}>1.5756 \mathrm{~Hz}$

Digamma diphoton excess

$750 \mathrm{GeV}$ (Petersson, 2016) > 181,349,201,089,887.59 THz >1.2886 THz > alg. $1.3031 \mathrm{Thz}>$ delta $1.12 \%$ >alg ref $1.1852 \mathrm{~Hz}>1.1720 \mathrm{~Hz}$

Gluon

$0.88 \mathrm{GeV}$ (Burgio, 2009) > 212,783,062,612.13 THz > 1.5480 Thz > ref $1.5549>$ delta $0.45 \%>$ ref. $1.4142 \mathrm{~Hz}>1.4078 \mathrm{~Hz}$

\section{Muon}

Muon $105 \mathrm{MeV}>25,388,888,152.58 \mathrm{THz}>1.4778 \mathrm{THz}>$ ref. $1.4660 \mathrm{THz}>$ delta $0.81 \%>$ ref. $1.3333 \mathrm{~Hz}>1.3441 \mathrm{~Hz}$

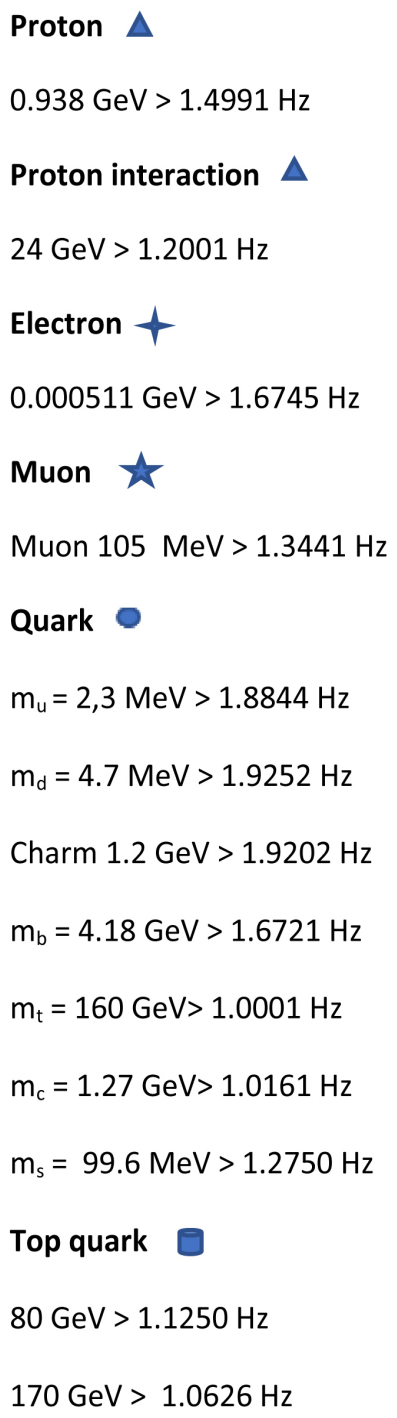

\section{Baryon}

$1650 \mathrm{MeV}>1.3201 \mathrm{~Hz}$ $3621 \mathrm{MeV}>1.4486 \mathrm{~Hz}$ $938.27 \mathrm{MeV}>1.5012 \mathrm{~Hz}$ Lepton

$0.5109 \mathrm{MeV}>1.6745 \mathrm{~Hz}$ $100.8 \mathrm{GeV}>1.260 \mathrm{~Hz}$ $1776.86 \mathrm{MeV}>1.4216 \mathrm{~Hz}$ Neutrino

$15.5 \mathrm{MeV}>1.5873 \mathrm{~Hz}$ $45.0 \mathrm{GeV}>1.1250 \mathrm{~Hz}$ $39.5 \mathrm{GeV}>0.9875 \mathrm{~Hz}$ $80.5 \mathrm{GeV}>1.0063 \mathrm{~Hz}$

\section{Boson}

$91.1875 \mathrm{GeV}>1.1399 \mathrm{~Hz}$ $80.385 \mathrm{GeV}>1.0049 \mathrm{~Hz}$ $270 \mathrm{GeV}>1.6875 \mathrm{~Hz}$

\section{Graviton}

$7.710^{-23} \mathrm{eV} / \mathrm{c}^{2}>1.2495 \mathrm{~Hz}$ 


\author{
$160 \mathrm{GeV} \gg>1.0001 \mathrm{~Hz}$ \\ $171 \mathrm{GeV}>1.0688 \mathrm{~Hz}$ \\ $1.3 \mathrm{GeV}>1.0401 \mathrm{~Hz}$ \\ Tetraquark \\ $4430 \mathrm{MeV}>1.7721 \mathrm{~Hz}$ \\ Pentaquark \\ $4.45 \mathrm{GeV}>1.7801 \mathrm{~Hz}$ \\ $4.39 \mathrm{GeV}>1.7561 \mathrm{~Hz}$
}

\section{Meson}

$139.57018 \mathrm{MeV}>1.7867 \mathrm{~Hz}$

Higgs

$126 \mathrm{GeV}>1.5756 \mathrm{~Hz}$

Digamma diphoton excess

$750 \mathrm{GeV}>1.1720 \mathrm{~Hz}$

Gluon

$0.88 \mathrm{GeV}>1.4078 \mathrm{~Hz}$

\section{Appendix 2. Zero-Point Energies of Elements}

Hydrogen $0.258 \mathrm{eV}$ (Gross, 1998): $62.38 \mathrm{Thz}$, delta 0.27\%

$\mathrm{D}_{2}: 0.185 \mathrm{eV}$ (Gross, 1998): $44.732 \mathrm{THz}$, delta $0.45 \%$

$\mathrm{O}_{2}: 787.380 \mathrm{~cm}^{-1}>23.60506 \mathrm{THz}$, delta $0.64 \%$ (Irikura, 2007)

$\mathrm{N}_{2}: 1175.78 \mathrm{~cm}^{-1} 35.24900 \mathrm{THz}$, delta 0.19\% (Irikura, 2007)

$\mathrm{C}_{2}: 924.0 \mathrm{~cm}^{-1} 27.70082 \mathrm{THz}$, delta $0.37 \%$ (Irikura, 2007)

OH: $1850.69 \mathrm{~cm}^{-1} 55.48229 \mathrm{THz}$, delta $0.22 \%$ (Irikura, 2007)

$\mathrm{P}_{2}: 389.70 \mathrm{~cm}^{-1} 11.68291 \mathrm{THz}$, delta $0.38 \%$ (Irikura, 2007)

SiO: $619.3921718 .56891 \mathrm{THz}$, delta $0.23 \%$

Appendix 3. Calculated Examples of Coherent Frequencies from Sub Hertz till PHz

\begin{tabular}{|c|c|c|c|c|c|c|c|c|c|c|c|c|}
\hline Factor & F1,m & F2,m & F3,m & F4,m & F5,m & F6,m & F7,m & F8,m & F9,m & F10,m & F11,m & F12,m \\
\hline $\mathrm{m}=0$ & 1.0000 & 1.0535 & 1.1250 & 1.1852 & 1.2656 & 1.3333 & 1.4142 & 1.5000 & 1.5803 & 1.6875 & 1.7778 & $1.8984 \mathrm{~Hz}$ \\
\hline $\mathrm{m}=1$ & 2.0000 & 2.1070 & 2.2500 & 2.3704 & 2.5312 & 2.6666 & 2.8284 & 3.0000 & 3.1606 & 3.3750 & 3.5556 & $3.7968 \mathrm{~Hz}$ \\
\hline $\mathrm{m}=2$ & 4.0000 & 4.2140 & 4.5000 & 4.7408 & 5.0624 & 5.3332 & 5.6568 & 6.0000 & 6.3212 & 6.7500 & 7.1112 & $7.5936 \mathrm{~Hz}$ \\
\hline $\mathrm{m}=3$ & 32.000 & 33.712 & 36.000 & 37.9264 & 40.4992 & 42.6656 & 45.2544 & 48.000 & 50.5696 & 54.000 & 56.8896 & $60.7488 \mathrm{~Hz}$ \\
\hline $\mathrm{m}=8$ & 256.00 & 269.70 & 288.00 & 303.41 & 324.00 & 341.33 & 362.04 & 384.00 & 404.54 & 432.00 & 455.12 & $486.00 \mathrm{~Hz}$ \\
\hline $\mathrm{m}=12$ & 4.0960 & 4.3151 & 4.6080 & 4.8546 & 5.1839 & 5.4613 & 5.7926 & 6.1440 & 6.4729 & 6.9120 & 7.2819 & $7.7759 \mathrm{KHz}$ \\
\hline $2^{\wedge} 24$ & 16.777 & 17.675 & 18.874 & 19.884 & 21.233 & 22.370 & 23.726 & 25.166 & 26.513 & 28.312 & 29.827 & $31.850 \mathrm{MHz}$ \\
\hline $2^{\wedge} 32$ & 4.2950 & 4.5248 & 4.8318 & 5.0904 & 5.4357 & 5.7266 & 6.0739 & 6.4425 & 6.7873 & 7.2478 & 7.6356 & $8.1536 \mathrm{GHz}$ \\
\hline $2^{\wedge} 40$ & 1.0995 & 1.1583 & 1.2370 & 1.3031 & 1.3915 & 1.4660 & 1.5549 & 1.6493 & 1.7376 & 1.8554 & 1.9547 & 2.0873 Thz \\
\hline $2^{\wedge} 48$ & 281.47 & 296.53 & 316.66 & 333.60 & 356.23 & 375.29 & 398.06 & 422.21 & 444.81 & 474.99 & 500.41 & 534.35 Thz \\
\hline & 532.5 & 505.6 & 473.4 & 449.3 & 420.8 & 399.5 & 376.6 & 710.1 & 674.0 & 631.3 & 599.1 & $561.0 \mathrm{~nm}$ \\
\hline
\end{tabular}

Appendix 4. Generalized Scale of Coherent Frequencies

$$
\mathrm{F}_{\mathrm{m}}(\text { coh. } 1)=2^{0} 3^{0} 2^{\mathrm{m}} \quad \mathrm{F}_{\mathrm{m}}(\mathrm{coh} .7)=2^{0.5} 2^{\mathrm{m}}
$$




$$
\begin{array}{ll}
\mathrm{F}_{\mathrm{m}}(\text { coh. } 2)=2^{8} 3^{-5} 2^{\mathrm{m}} & \mathrm{F}_{\mathrm{m}}(\text { coh. } 8)=2^{-1} 3^{1} 2^{\mathrm{m}} \\
\mathrm{F}_{\mathrm{m}}(\text { coh. } 3)=2^{-3} 3^{2} 2^{\mathrm{m}} & \mathrm{F}_{\mathrm{m}}(\text { coh. } 9)=2^{7} 3^{-4} 2^{\mathrm{m}} \\
\mathrm{F}_{\mathrm{m}}(\text { coh. } 4)=2^{5} 3^{-3} 2^{\mathrm{m}} & \mathrm{F}_{\mathrm{m}}(\text { coh. } 10)=2^{-4} 3^{3} 2^{\mathrm{m}} \\
\mathrm{F}_{\mathrm{m}}(\text { coh. } 5)=2^{-6} 3^{4} 2^{\mathrm{m}} & \mathrm{F}_{\mathrm{m}}(\text { coh. } 11)=2^{4} 3^{-2} 2^{\mathrm{m}} \\
\mathrm{F}_{\mathrm{m}}(\text { coh. } 6)=2^{2} 3^{-1} 2^{\mathrm{m}} & \mathrm{F}_{\mathrm{m}}(\text { coh. } 12)=2^{-7} 3^{5} 2^{\mathrm{m}}
\end{array}
$$

\section{Appendix 5. Generalized Scale for Non-Coherent}

\section{Frequencies}

$$
\begin{aligned}
& \mathrm{D}_{\mathrm{m}}(\text { decoh. } 1)=10^{(0.5 \operatorname{logF} 1+0.5 \log \mathrm{F} 2)} \quad \mathrm{D}_{\mathrm{m}}(\text { decoh. } 2)=10^{(0.5 \log 2+0.5 \operatorname{logF} 3)} \\
& \mathrm{D}_{\mathrm{m}}(\text { decoh. } 3)=10^{(0.5 \log F 3+0.5 \log F 4)} \quad \mathrm{D}_{\mathrm{m}}(\text { decoh. } 4)=10^{(0.5 \log F 4+0.5 \log 55)} \\
& \mathrm{D}_{\mathrm{m}}(\text { decoh. } .5)=10^{(0.5 \log \mathrm{F} 5+0.5 \log \mathrm{F} 6)} \quad \mathrm{D}_{\mathrm{m}}\left(\text { decoh.6) }=10^{(0.5 \log \mathrm{F} 6+0.5 \log \mathrm{F} 7)}\right. \\
& \mathrm{D}_{\mathrm{m}}(\text { decoh. } .)=10^{(0.5 \log \mathrm{F} 7+0.5 \log \mathrm{F} 8)} \quad \mathrm{D}_{\mathrm{m}}(\text { decoh. } 8)=10^{(0.5 \log \mathrm{F} 8+0.5 \log F 9)} \\
& \mathrm{D}_{\mathrm{m}}(\text { decoh.9 })=10^{(0.5 \log \mathrm{F} 9+0.5 \log \mathrm{F} 10)} \quad \mathrm{D}_{\mathrm{m}}(\text { decoh. } 10)=10^{(0.5 \operatorname{logF} 10+0.5 \operatorname{logF} 11)} \\
& \mathrm{D}_{\mathrm{m}}(\text { decoh. } 11)=10^{(0.5 \log F 11+0.5 \log \mathrm{F} 12)} \quad \mathrm{D}_{\mathrm{m}}(\text { decoh.12 })=10^{(0.5 \log \mathrm{F} 12+0.5 \log \mathrm{F} 13)}
\end{aligned}
$$

